\title{
In situ Reaction Monitoring in Heterogeneous Catalysts by a Benchtop NMR Spectrometer
}

\author{
Markus Leutzsch, Andrew J. Sederman, Lynn F. Gladden, Michael D. \\ Mantle* \\ Department of Chemical Engineering and Biotechnology, Philippa Fawcett Drive, \\ Cambridge, CBз OAS, UK
}

\begin{abstract}
Understanding the reactivity and mass transport properties of porous heterogenous catalysts is important for the development of new materials. Whereas MRI has previously been used to correlate chemical kinetics and hydrodynamics under operando conditions, this paper demonstrates that a modern benchtop NMR spectrometer is a suitable alternative to obtain diverse reaction information in porous heterogeneous catalyst materials on a smaller scale. Besides information about the chemical conversion within the pores, it can also be used to study changes of surface interaction by $T_{1} / T_{2}$ NMR relaxometry techniques and changes in mass transport by PFG NMR from a single chemical reaction.
\end{abstract}

Keywords: heterogeneous catalysis, benchtop NMR, reaction kinetics, porous materials, NMR relaxometry

\section{Introduction}

Many industrially relevant processes in bulk and fine chemical, and pharmaceutical industries use porous, heterogeneous materials[1]. In contrast to reactions catalysed by a homogeneous catalyst in the liquid phase, where the rate determining step is often controlled at a molecular level, heterogeneous reactions within porous materials are more complex as mass transport of reactants and products between and within pores, to the active catalytic centre

\footnotetext{
*Corresponding author

Email address: mdm20@cam.ac.uk (Michael D. Mantle)
} 
or to an external surface often become rate limiting steps in these systems. In reactors with heterogeneous catalysts the dispersion and flow hydrodynamics are additional important parameters. Magnetic resonance imaging (MRI) techniques have been utilised to study such chemical reactors under operando conditions and deliver hydrodynamic and chemical information from a single reactor that are difficult to obtain from other techniques [2 4]. Information about chemical conversion is mostly obtained from ${ }^{1} \mathrm{H}$ NMR data and therefore limited to simple systems, where the chemical shifts of reactants and products are well separated. Nevertheless, this method was successfully applied to obtain spatially resolved conversion information for industrially relevant processes like the esterification of acetic acid[5], the hydrogenation of $\alpha$-methyl styrene [6] and ethylene oligomerisation [7, 8]. For more complex reactions, where multiple components overlap, ${ }^{13} \mathrm{C}$ detection can significantly improve the separation due to the increased chemical shift dispersion, but it comes at the price of increased acquisition time and reduced time resolution due to the low natural abundance of ${ }^{13} \mathrm{C}(1.1 \%)$. With polarisation techniques, like DEPT[9], the acquisition time can be reduced without the need of the expensive ${ }^{13} \mathrm{C}$ enriched samples. Spatially resolved DEPT has previously been used to obtain information about isomerisation processes during 1-octene hydrogenation [10] or selectivity preferences of competitive etherification and hydration reactions of 2-methyl-2-butene[11]. Besides a knowledge of the correlation of hydrodynamic and chemical kinetics in a chemical reactor, understanding the properties of the heterogeneous catalyst is important to improve industrially relevant processes and to develop new and improved materials. NMR relaxation techniques have recently been shown to examine solvent effects [12], reactivity trends, and adsorption strengths in porous, catalytic systems[13, 14]. In recent years, benchtop NMR systems with medium magnetic field $\left(B_{0}\right)$ strengths between 1 and 2 Tesla and high $B_{0}$ field homogeneity $(<0.03 \mathrm{ppm})$ have become widely available on the market. Due to their low price, small physical footprint and minimal maintenance requirements these magnets are receiving growing interest in academia and industry [15-17]. In addition to classic spectroscopy and relaxometry measurements, some benchtop systems also allow the investigation of mass transport phenomena by PFG NMR. Besides their value for teaching purposes [18 20], they have been widely utilised for in- and on-line measurements of homo- and heterogeneous reactions in flow and batch systems $21-25]$ and fermentation processes [26]. In contrast to the previously described high field MRI studies, reports that utilised a benchtop NMR instrument to inves- 
tigate heterogeneous reactions, only used it as an ex situ sampling technique. Herein we describe a different approach by using a benchtop NMR spectrometer to study a heterogenous catalytic reaction, which combines spectroscopic techniques for composition information with relaxometry and diffusometry measurements to study adsorption and mass transport phenomena.

\section{Materials and Methods}

\subsection{Catalyst Synthesis}

The heterogeneous $0.3 \mathrm{wt} \% \mathrm{Pd} / \mathrm{TiO}_{2}$ catalyst was prepared by incipient wet impregnation. The catalyst support material used were $\mathrm{TiO}_{2}$ extrudate pellets (Johnson Matthey, UK, $3.3 \mathrm{~mm}$ cylindrical diameter with an average length of $4-6 \mathrm{~mm}$ ). The pore volume was determined to be $0.42 \mathrm{ml}$ water per $1 \mathrm{~g} \mathrm{TiO} 2$ by averaging 10 measurements of individual pellets. The pellets were saturated by adding an aqueous $\mathrm{Pd}\left(\mathrm{NO}_{3}\right)_{2} \cdot 2 \mathrm{H}_{2} \mathrm{O}$ solution $(17.88 \mathrm{mg} / \mathrm{ml}$, Strem Chemicals UK Ltd, Cambridge, UK) to the pellets. Oven-drying for $12 \mathrm{~h}$ at $130{ }^{\circ} \mathrm{C}$, followed by calcination at $420{ }^{\circ} \mathrm{C}$ for $3 \mathrm{~h}$ under nitrogen flow in a furnace provided the non-activated $0.3 \mathrm{wt} \% \mathrm{Pd} / \mathrm{TiO}_{2}$ catalyst. Prior to the NMR experiments the $0.3 \mathrm{wt} \% \mathrm{Pd} / \mathrm{TiO}_{2}$ was activated under flowing hydrogen $(20 \mathrm{~mL} / \mathrm{min})$ for $5-10 \mathrm{~min}$ until the catalyst pellets turned black indicating the reduction of $\mathrm{PdO}$ to $\mathrm{Pd}(0)$ and $\mathrm{H}_{2} \mathrm{O}$. The catalyst was then dried for $24 \mathrm{~h}$ at $100{ }^{\circ} \mathrm{C}$ to remove adsorbed hydrogen and water that was formed during the catalyst activation.

\subsection{NMR Experiments}

High field NMR data were acquired on a Bruker AVANCE III HD NMR spectrometer (Bruker BioSpin GmbH, Rheinstetten, Germany) operating at a ${ }^{1} \mathrm{H}$ resonance frequency of $299.84 \mathrm{MHz}$ equipped with a $10 \mathrm{~mm}{ }^{1} \mathrm{H}$ selective probe $(\pi / 2$ pulse length $=10 \mu \mathrm{s})$. The benchtop NMR experiments were performed using an Oxford Instruments Pulsar NMR Spectrometer (Oxford Instruments, Abingdon, UK) at $37^{\circ} \mathrm{C}$ operating at a ${ }^{1} \mathrm{H}$ resonance frequency of $59.7 \mathrm{MHz} .{ }^{1} \mathrm{H}$ NMR spectra were acquired after applying a $\pi / 2$ pulse $(12 \mu \mathrm{s})$ and a relaxation delay of $10 \mathrm{~s}$ in a single scan. $T_{2}$ data were obtained from averaging $4 \mathrm{CPMG}$ echo trains with 5000 echo points separated by a echo spacing $\tau=600 \mu \mathrm{s}$. $T_{1}-T_{2}$ relaxation correlation data were obtained by incrementing the inversion delay in an inversion recovery sequence prior to a CPMG echo train. The $2 \mathrm{D}$ data are inverted numerically as reported previously [13, 14] to form a $2 \mathrm{D}$ distribution of $T_{2}$ correlated against 
$T_{1}$. ${ }^{1} \mathrm{H}$ PFG NMR measurements were performed using the z-shim coil of the Pulsar spectrometer capable of producing a field of approximately 250 $\mathrm{mT} / \mathrm{m}$. The PFG data in the heterogeneous pellets were obtained with an alternated pulsed-field gradient stimulated echo (AGPSTE) sequence 27] in order to minimise the effect of internal magnetic field gradients. The gradient strength was calibrated using a reference self-diffusion coefficient $D=3.04 \times 10^{-9} \mathrm{~m}^{2} \mathrm{~s}^{-1}$ of water at $37^{\circ} \mathrm{C}[28$. The diffusion value was obtained after fitting the signal attenuation to the following equation[27]:

$$
I(g)=I_{0} e^{-(\gamma g \delta)^{2}(\Delta+3 \tau / 2-\delta / 12) D},
$$

where $I_{0}$ and $I(g)$ are is the NMR integral in the absence and presence of at a specific gradient strength $g$ respectivly, $\gamma$ is the proton gyromagnetic ratio and $D$ is the self diffusion coefficient of the component of interest. The total time of the dephasing gradient $\delta$ was $3 \mathrm{~ms}$ and the diffusion time $\Delta$ was $75 \mathrm{~ms}$ in all the reported measurements. The time after the initial $\pi / 2$ and the first $\pi$ pulse $\tau$ was $5.6 \mathrm{~ms}$. A gradient recovery delay of $2 \mathrm{~ms}$ was used. NMR data were processed and plotted with MNova 12.02 (Mestrelab Research, S.L., Santiago de Compostela, Spain). For fitting reaction data and plotting of graphs MATLAB (R2016b, The MathWorks, MA, USA) was used.

\subsection{Reaction Monitoring}

An activated catalyst pellet (55 mg, length $5 \mathrm{~mm}$ ) was soaked in 1-octene $(97+\%$, Alfa Aesar, Heysham, UK) for $30 \mathrm{~min}$. After this time no air bubble formation was observed indicating that the pellets are fully saturated. After drying the outer surface of the pellet with filter paper, the soaked catalyst material was placed in a $5 \mathrm{~mm}$ o.d. thin wall NMR tube reactor (i.d. $=4.09$ $\mathrm{mm}$, Wilmad-LabGlass, Vineland, USA), and positioned in the centre of the NMR coil. The reaction was initiated by flowing hydrogen (Air Liquide UK Ltd., Birmingham, UK) with a flow rate of $8 \mathrm{ml} / \mathrm{min}$ above the sample. ${ }^{1} \mathrm{H}$ spectroscopic and CPMG $T_{2}$ data were acquired once every $60 \mathrm{~s}$ until no further conversion was observed. Magnetic field drifts were corrected by automatically changing the offset to the highest peak in the NMR spectrum.

\section{Results and Discussion}

${ }^{1} \mathrm{H}$ NMR signals of liquids in porous materials like heterogeneous catalysts are often broadened due to magnetic susceptibility differences on the 
interfaces of liquids within pores, particles and surfaces or due to different orientations of solid catalyst material within a reactor. A representative high field $(7 \mathrm{~T}){ }^{1} \mathrm{H}$ NMR spectrum of such a case is shown in figure 1. The spectrum was obtained for 1-octene in $\mathrm{TiO}_{2}$ pellets, a common heterogeneous catalyst support [29]. Two clearly distinct aliphatic $\left(\delta_{1_{\mathrm{H}}}=0-2 \mathrm{ppm}\right)$ and olefinic chemical shift regions $\left(\delta_{1} \mathrm{H}=4.5-6.5 \mathrm{ppm}\right)$ were observed and could be directly assigned to the alkyl chain and the double bond in the molecule respectively. The methylene group next to the $\mathrm{C}-\mathrm{C}$ double bond and the terminal methyl group only appear as shoulders from the signal of the internal methylene protons. The olefinic region has two distinct peaks at 4.9 and $5.8 \mathrm{ppm}$. However, quantitation is complicated due to the excessive overlap of those peaks. When reducing the sample size from $10 \mathrm{~mm}$ to $5 \mathrm{~mm}$ NMR tubes and orienting all the pellets along the same axis, the spectral resolution is improved as seen in figure 1 .

The olefinic proton signals of the terminal $\mathrm{CH}_{2}$ group $\left(\delta_{1_{\mathrm{H}}}=4.9 \mathrm{ppm}\right)$ and the internal $\mathrm{CH}$ group $\left(\delta_{1_{\mathrm{H}}}=5.8 \mathrm{ppm}\right)$ are now separated at $30 \%$ of the maximum height of the peaks, which makes integration easier and enables the observation of changes between them. Similar effects are visible in the aliphatic part of the spectrum, where all three proton environments are visible as clear peaks rather then shoulders. When measuring the $5 \mathrm{~mm}$ sample in a $1.4 \mathrm{~T}$ benchtop NMR system, the proton spectrum obtained at lower field look surprisingly similar. In comparison with spectra of bulk liquids at those fields, the differences in the $\mathrm{TiO}_{2}$ pellets are small. The splitting obtained from the olefinic spin-spin coupling, $J_{\text {cis }}=10 \mathrm{~Hz}$ and $J_{\text {trans }}=17 \mathrm{~Hz}$, correspond to $0.17 \mathrm{ppm}$ and $0.28 \mathrm{ppm}$ at $59.7 \mathrm{MHz}$ and $0.03 \mathrm{ppm}$ and 0.06 ppm at $299.84 \mathrm{MHz}$ respectively. In porous materials this difference becomes less significant as it is compensated by the lower magnetic susceptibility line broadening effect for the liquid in the porous $\mathrm{TiO}_{2}$ at the medium field. In the current example, the coupling difference in ppm due to different $B_{0}$ fields is still visible for the internal olefinic proton. The aliphatic signals from the samples are also comparable, although the lines are slightly broader for the medium field instrument. It is worth mentioning that all the spectra were acquired in less then a minute and the signal-to-noise obtained from all of them is sufficient as seen in figure 1. The small differences between the NMR spectra obtained at different field strengths raises the question whether one could use a benchtop NMR machine to investigate a reacting system. Therefore, the hydrogenation of 1-octene to octane in a $0.3 \mathrm{wt} \% \mathrm{Pd} / \mathrm{TiO}_{2}$ was chosen as an example to test this potential. Proton NMR spectra obtained 


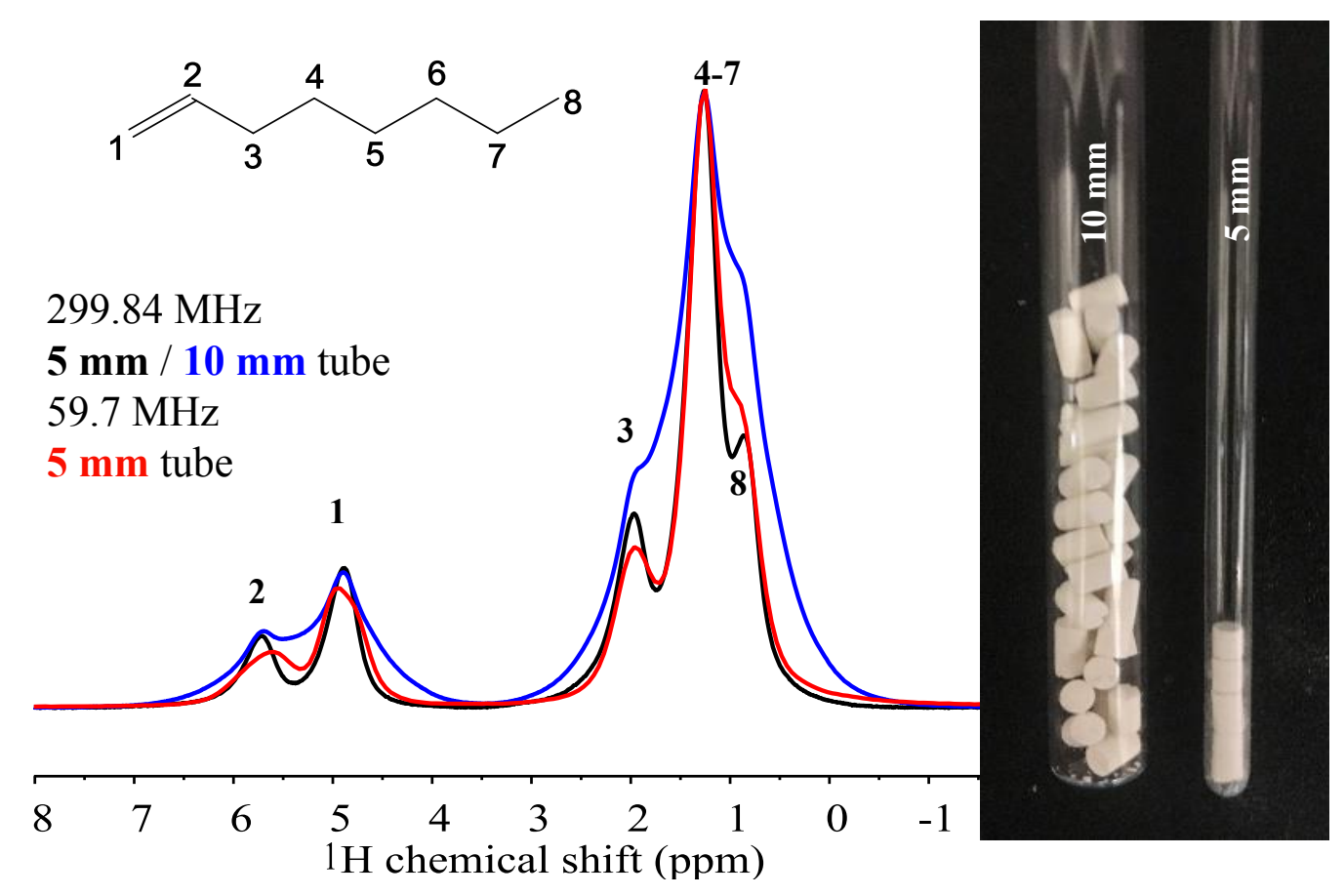

Figure 1: ${ }^{1} \mathrm{H}$ NMR spectra of 1-octene in a heterogeneous $\mathrm{TiO}_{2}$ support acquired at 59.7 MHz (1.4 T) (-) and $299.84 \mathrm{MHz}(7 \mathrm{~T})$ in a $5 \mathrm{~mm} \mathrm{(-)}$ and $10 \mathrm{~mm}$ NMR tube (-). The chemical structure of 1-octene is shown on the top left and the corresponding assignments are shown above the peaks in the spectra. The right image shows the respective $5 \mathrm{~mm}$ and $10 \mathrm{~mm}$ sample used for the acquisition of the data. 

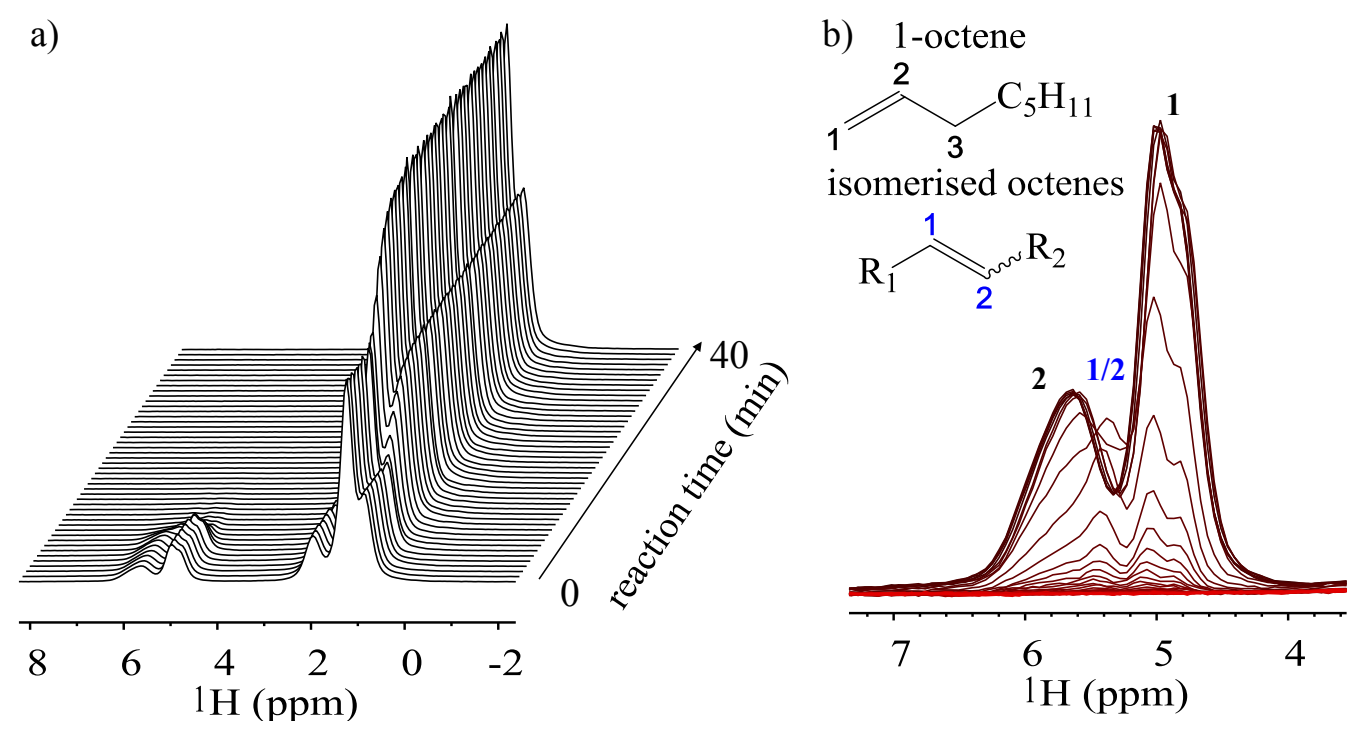

Figure 2: ${ }^{1} \mathrm{H}$ NMR spectra acquired every minute after the hydrogen flow was started. a) ${ }^{1} \mathrm{H}$ NMR spectra indicating the overall conversion from the alkene to the alkane; b) Olefinic region $(4.5-6.5 \mathrm{ppm})$ indicating isomerisation processes from 1 -octene to cis/trans-2/3octenes.

at different time points of the hydrogenation reaction are shown in figure 2 . The acquisition of NMR data began once the hydrogen flow was initiated. After an induction period of approximately seven minutes during which the air in the setup was replaced by hydrogen, the reaction started. Two different processes can be observed in the ${ }^{1} \mathrm{H}$ NMR spectrum: the reduction of the $\mathrm{C}=\mathrm{C}$ double bond and the isomerisation of 1-octene to internal octenes, as shown in figure $2 \mathrm{~b}$. It is remarkable that the isomerisation process can be seen at this medium field from ${ }^{1} \mathrm{H}$ NMR data. Previously ${ }^{13} \mathrm{C}$-DEPT MRI was used to differentiate between those two processes as the signal separation was not sufficient enough to obtain this information of a hydrogenation reaction in $\mathrm{Pd} / \mathrm{Al}_{2} \mathrm{O}_{3}$ from ${ }^{1} \mathrm{H}$ NMR spectroscopy [10. In the current ${ }^{1} \mathrm{H}$ NMR study it is not possible to distinguish between the double bond geometry (cis or trans) and the exact position of the internal double bond (2, 3 or 4). However, when one is interested in the properties of a heterogeneous catalyst it is more important to see whether isomerisation occurs or not. The observation of the isomerisation process also enables the deconvolution of the isomerisation rate and reduction rate. By comparing the signal of the external $\mathrm{CH}_{2}$ protons against the total olefinic signal region, one can obtain quantitative 

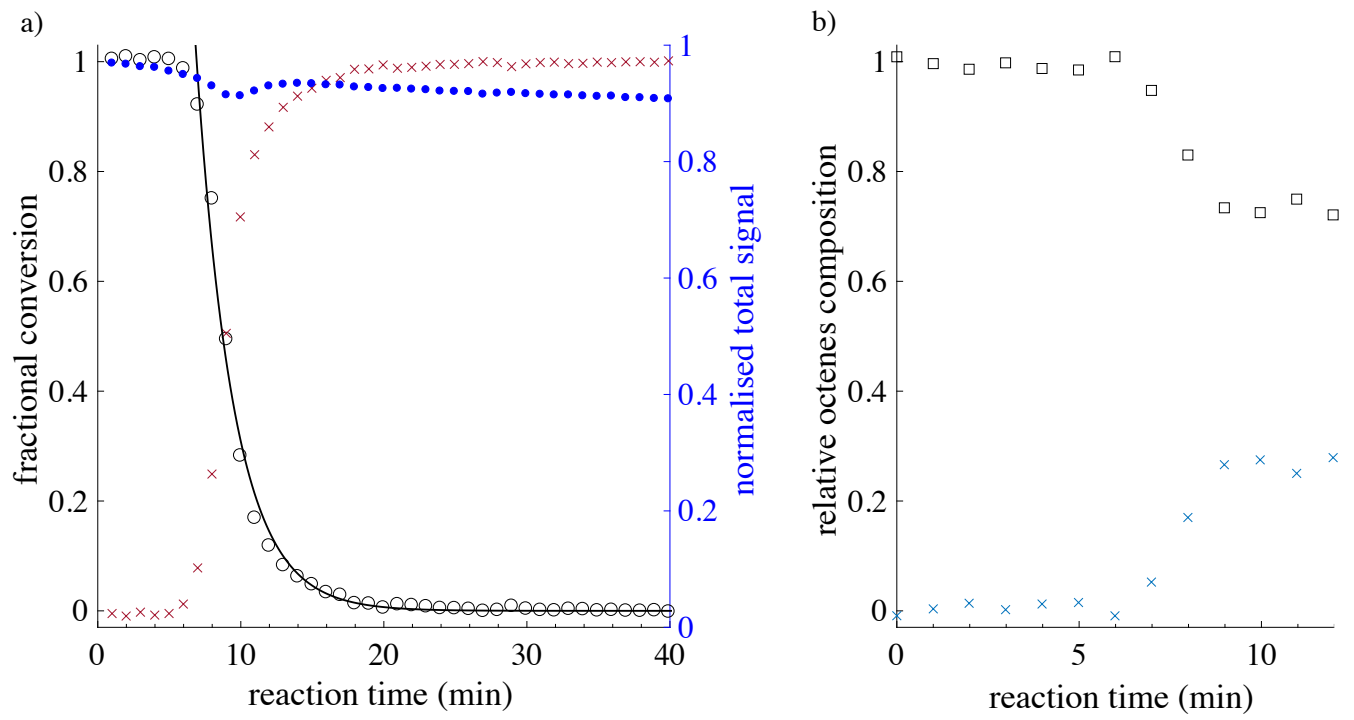

Figure 3: a) Fractional conversion of octenes $(\bigcirc)$ to octane $(\times)$ in $0.3 \mathrm{wt} \% \mathrm{Pd} / \mathrm{TiO}_{2}$ is described by the left axis. The black line $(-)$ shows the fit to a first order exponential rate. The normalised total NMR signal before $(*)$ and after conversion correction $(\bullet)$ are described by the right axis. b) Isomerisation from 1-octene $(\square)$ to $2 / 3$-octene $(\times)$ observed during the reduction of 1-octene to octane in $0.3 \mathrm{wt} \% \mathrm{Pd} / \mathrm{TiO}_{2}$.

data of the isomerisation process. The results are shown in figure 3. Both processes proceed in parallel. On a molecular scale this is not surprising. After the formation of a half-hydrogenated alkene intermediate on the Pd surface, two different reaction pathways are possible: 1) reductive elimination delivers the desired alkane; 2) $\beta$-hydride elimination gives the isomerised olefins. Once octane is formed the reaction is irreversible at room temperature. The overall alkene conversion is complete approximately 20 minutes after the first reaction was observed. The conversion can be described by a first order exponential reaction $\left(A=A_{0} e^{-k t}\right)$ with a rate constant $k=0.38 \pm 0.05 \mathrm{~min}^{-1}$. In first order rate reactions the rate determining step is dependent on the concentration of one reactant. As there is a constant supply or hydrogen from the gas phase in our system, the limiting reactant is the olefin. The more alkene that is converted, the lower the probability that a reactive molecule reaches the active catalytic site and therefore the reaction rate decreases. The isomerisation can be followed until approximately $70 \%$ conversion is reached. Afterwards the signal-to-noise ratio is too low to obtain reliable data. The ratio between 1-octene and isomerised octenes reaches a steady-state four 
minutes after the reaction started. This equilibrium is dependent on a different factors, for example: 1) individual formation rates of the isomers; 2) the reactivity of the intermediates towards reduction; 3) adsorption strength of the isomers on the surface. When developing new materials and catalysts it is important to obtain information about the extent of these processes as they can dramatically influence the reaction kinetics and the yields of the desired products. The overall NMR signal as shown in figure $3 \mathrm{a}$ is increasing during the reaction due to the hydrogen addition into the alkene. After correcting the total signal to compensate the chemical conversion, two other effects become visible: 1) a short signal intensity decrease after the reaction started; 2) a constant drop of signal during the whole measurements. The first drop of signal can be explained by the exothermic hydrogenation reaction and the change of the Boltzmann distribution. A reaction temperature increase of $5^{\circ} \mathrm{C}$ can already decrease the equilibrium magnetisation by $2 \%$. Once the reaction reaches higher conversion, this effect becomes smaller as the system thermally equilibrated back to the magnet temperature $\left(37^{\circ} \mathrm{C}\right)$. The constant signal drop during the whole experiment is likely due to liquid evaporation and the gas flow. As the amount of liquid decreases only around $3 \%$ during the reaction, is not significant enough to have a big influence on the reaction rate. Although, when investigating slower reactions in a batch setup, this process might become an important factor.

In addition to the classic spectroscopic approach to follow the conversion by a simple pulse acquire experiment, the heterogeneous reaction can also be studied by following changes in the $T_{2}$ distribution by measuring a CPMG echo train. After each proton acquisition, a $T_{2}$ distribution was obtained from a CPMG measurement. In order to obtain comparable time-resolved $T_{2}$ reaction data, the CPMG decays were subsequently inverted by inverse Laplace transformation with a fixed $\alpha$-value during the regularisation. The results are shown in figure 4 . Before the reaction started, there are two different major $T_{2}$ components, with $T_{2,1}=125 \mathrm{~ms}$ and $T_{2,2}=322 \mathrm{~ms}$, observed. A potential explanation for these two populations is that an increased surface interaction of the $\mathrm{C}-\mathrm{C}$ double-bond slows down the exchange between the surface and bulk species, so that the rapid two-phase exchange model [30, 31] does not apply and two individual species can be obtained. Alternatively, the enhanced surface interaction can slow down the exchange between pores of different size and lead to different relaxation regimes. Once the reaction started only one broad $T_{2}$ peak is observed, which can be correlated with the 


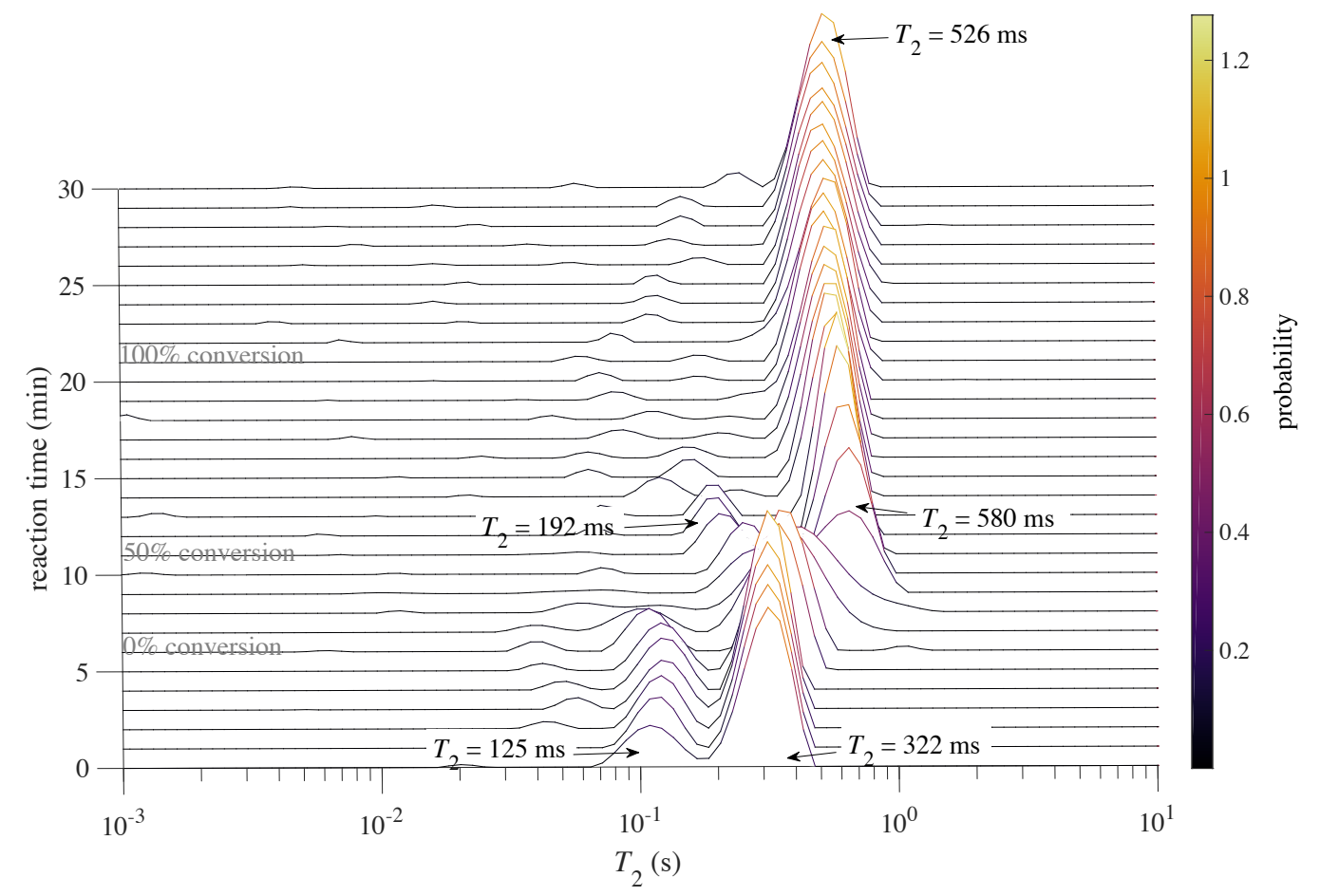

Figure 4: $T_{2}$ distributions obtained after Laplace inversion at different time points during the hydrogenation of 1-octene in $0.3 \mathrm{wt} \% \mathrm{Pd} / \mathrm{TiO}_{2}$. 
high reaction rate and signal averaging over the measurements at this time point. Once the reaction reached about $50 \%$ conversion, two major species can be observed again with $T_{2,3}=192 \mathrm{~ms}$ and $T_{2,4}=580 \mathrm{~ms}$. In this case the first could be connected to unreacted olefin, that spends a higher average time on the surface and the latter with the newly formed alkane that is less interacting and therefore has a longer relaxation time. Upon reaction completion, only one dominant $T_{2}$ component with $T_{2,5}=526 \mathrm{~ms}$ is obtained. As the product is expected to interact less with the surface, the observation of only one averaged major species suggests that it follows the rapid two-phase fast exchange model. These time-resolved in situ $T_{2}$ measurements in combination with the classical spectroscopy data are quite unique and enable the study of reaction kinetics and change of surface properties. Low field NMR machines $\left(B_{0}<0.5 \mathrm{~T}\right)$ generally do not have the spectral resolution to investigate complex reactions and high field spectrometers $\left(B_{0}>4 \mathrm{~T}\right)$ are limited by their increased internal magnetic field gradient effect in porous material and power limitations for acquiring CPMG echo trains. Medium field benchtop NMR systems are a good compromise to obtain information from both, spectroscopy and relaxometry. Other measurements on the heterogeneous system were performed to further show the potential of medium field spectrometers to investigate reactions in porous materials. In order to understand changes in surface interactions during the reaction, $T_{1}-T_{2}$ correlation plots were acquired before and after the reaction and are shown in figure 5. In agreement with the kinetic CPMG data, two different $T_{2}$ regions are observed for 1-octene $\left(T_{1} / T_{2}=10\right.$ and $\left.T_{1} / T_{2}=2\right)$. Interestingly, both $T_{1}$ values are within the same order of magnitude. Only one peak $\left(T_{1} / T_{2}=2.6\right)$ is obtained from octane. When comparing this value to the two ratios of 1-octene this data suggests, in correlation with previous reports [13, 14], that one species $\left(T_{1} / T_{2}=10\right)$ has a higher affinity to the surface, whereas the other $\left(T_{1} / T_{2}=2\right)$ has a lower surface interaction compared to the product. Generally, one would expect that the alkane has a lower surface affinity than the alkene due to the missing $\mathrm{OH}-\pi$ or metal $-\pi$ interactions between the $\mathrm{C}=\mathrm{C}$ double bond and hydroxyl groups or the palladium on the surface of the catalyst. When recalling the previously proposed hypothesis that one of the two alkene species is more influenced by the surface interaction, whereas the other component is more dominated by the bulk part, this observation becomes more understandable. The alkane peak instead represents the overall average of all species in the porous material. Another common parameter of interest for heterogeneous reactions are mass transport properties of the 


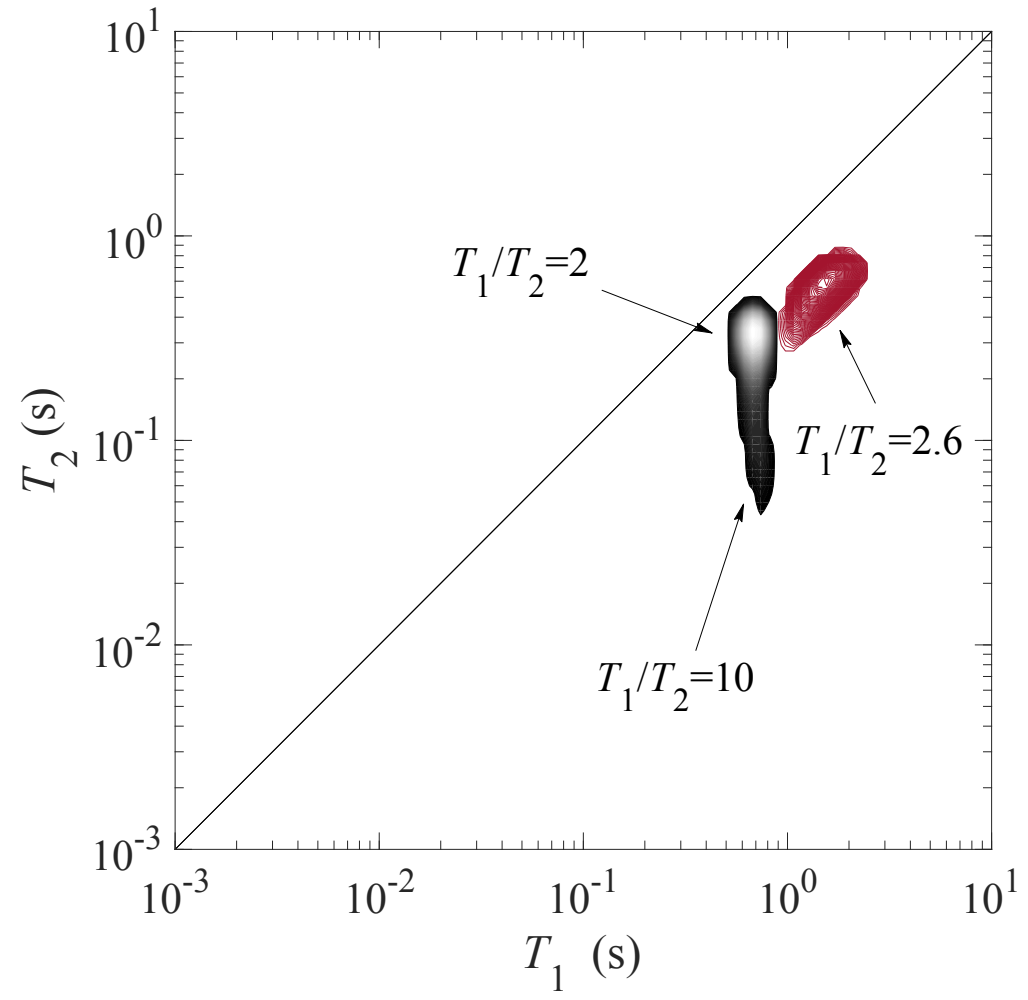

Figure 5: $T_{1}-T_{2}$ relaxation correlation plots obtained from 1-octane before (๑) and noctane (๑) after the reaction in $0.3 \mathrm{wt} \% \mathrm{Pd} / \mathrm{TiO}_{2}$ was completed. The diagonal line represents $T_{1} / T_{2}=1$. 


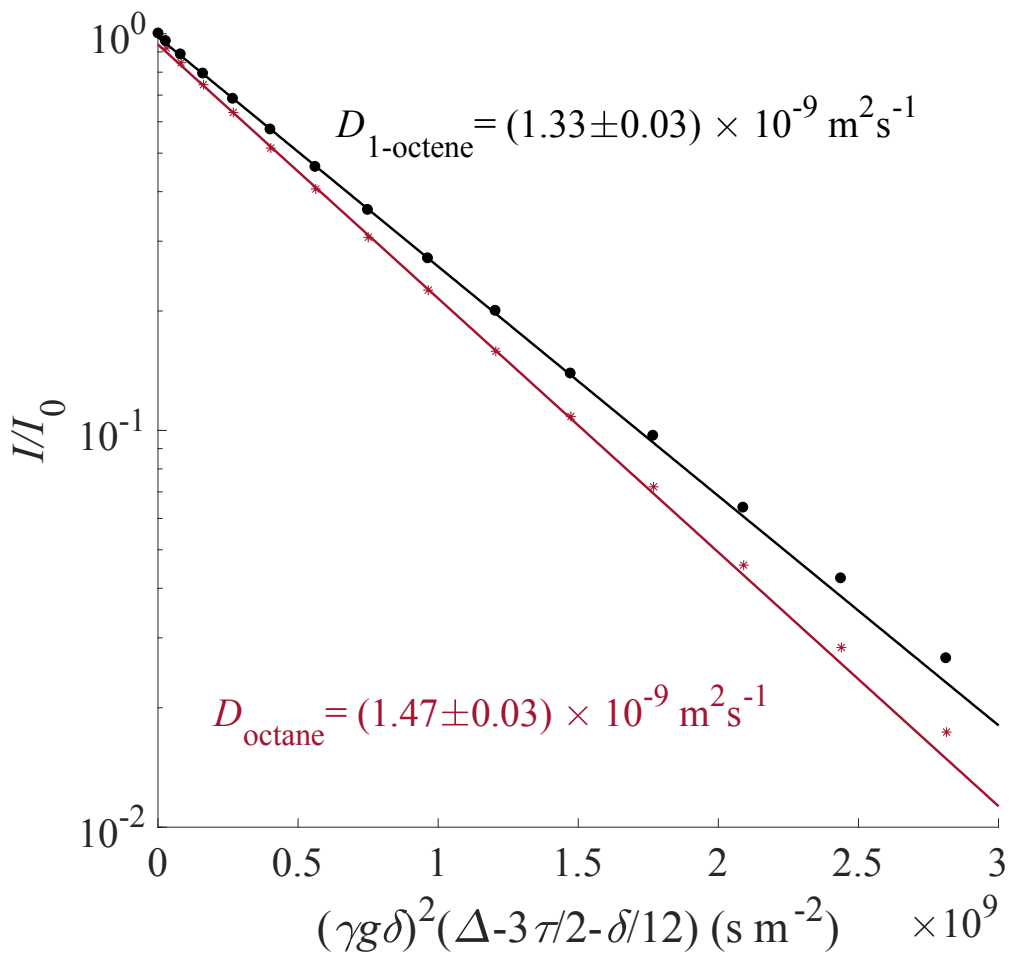

Figure 6: AGPSTE attenuation plots obtained from 1-octane $(\bullet)$ before and n-octane $(*)$ after the hydrogenation reaction in $0.3 \mathrm{wt} \% \mathrm{Pd} / \mathrm{TiO}_{2}$ was completed. Straight lines were obtained after fitting the data to equation 1 . The plotted self diffusion values are presented as obtained after fitting the data.

system obtained from PFG NMR studies. Due to the rapidx reaction, the self diffusion values of the reactant and products in the porous sample could only be investigated before and after the reaction. The results are presented in figure 6. The signals of the starting material and the product could be attenuated by two orders of magnitude. Both decays can be described by a single component diffusion, $D_{1 \text {-octene }}=(1.33 \pm 0.03) \times 10^{-9} \mathrm{~m}^{2} \mathrm{~s}^{-1}$ and $D_{\text {octane }}=(1.47 \pm 0.03) \times 10^{-9} \mathrm{~m}^{2} \mathrm{~s}^{-1}$. The self-diffusion coefficient is reduced compared to the bulk value due to catalyst tortuosity. The diffusion coefficients of 1-octene and octane are the same order of magnitude; however, the diffusion coefficient of 1-octene is approximately $10 \%$ smaller. Previous reports probed the surface diffusion of 1-octene in $\theta-\mathrm{Al}_{2} \mathrm{O}_{3}$ [32]. In our reported system such diffusion components were not observed. As they have a low population and slow diffusion coefficients, signal-to-noise limitations of 
the benchtop system and the low available gradient strengths of the instrument limit the chances of probing such a species. When investigating systems, where the difference of diffusivity of the starting material and the product is greater or where the mass transport of a substrate between particles plays an important role, this combined methodology has a great potential and it can enable the correlation between reactivity and mass transport phenomena from a simple benchtop NMR system.

\section{Conclusions}

${ }^{1} \mathrm{H}$ NMR spectra of liquids obtained from a medium field NMR magnet in a porous, heterogeneous catalyst material are able to compete with those obtained from high field machines due to reduced magnetic susceptibility line broadening effects. This was exploited to investigate the reduction of 1-octene in $0.3 \mathrm{wt} \% \mathrm{Pd} / \mathrm{TiO}_{2}$ as an example reaction. Besides the chemical conversion information to the desired octane, the extent of isomerisation could also be studied. Furthermore, time-resolved $T_{2}$ studies allowed changes in the surface interaction of the substrates with the porous material to be probed. Additionally, the benchtop NMR spectrometer was also used to investigate changes of surface interaction strength of reactants and products by measuring $T_{1} / T_{2}$ ratios and changes in mass transport from PFG NMR experiments. The high information content obtained from such a small sample can help to better understand newly developed catalyst materials in the future. Due to an increased mobility of those benchtop systems, they can also be used to combine NMR with other techniques, like total neutron scattering, as recently demonstrated [33. From these combined approaches one can obtain an even deeper insight into physicochemical processes occurring within pores and help to improve the optimisation of new heterogenous catalyst materials in the future.

\section{Acknowledgments}

The authors would like to thank funding from EPSRC (EP/N009304/1, Understanding Liquid Phase Heterogeneous Catalysis to Develop Catalytic Processes). Raw experimental NMR data may be accessed at https://doi. org/10.17863/CAM.26399. 


\section{References}

[1] G. Ertl, H. Knözinger, F. Schüth, J. Weitkamp, Handbook of Heterogeneous Catalysis, Wiley-VCH, Weinheim, 2008. doi:10.1002/ 9783527610044 .

[2] S. Stapf, S.-I. Han (Eds.), NMR Imaging in Chemical Engineering, Wiley-VCH Verlag GmbH \& Co. KGaA, Weinheim, FRG, 2005. doi: 10.1002/3527607560.

[3] L. F. Gladden, Magnetic resonance in reaction engineering: beyond spectroscopy, Curr. Opin. Chem. Eng. 2 (3) (2013) 331-337. doi: $10.1016 / j$. coche.2013.05.005.

[4] A. A. Lysova, I. V. Koptyug, Magnetic resonance imaging methods for in situ studies in heterogeneous catalysis, Chem. Soc. Rev. 39 (12) (2010) 4585. doi:10.1039/b919540h.

[5] E. Yuen, A. Sederman, L. Gladden, In situ magnetic resonance visualisation of the spatial variation of catalytic conversion within a fixed-bed reactor, Appl. Catal. A Gen. 232 (1-2) (2002) 29-38. doi: 10.1016/S0926-860X(02)00064-9.

[6] I. Koptyug, A. Lysova, A. Kulikov, V. Kirillov, V. Parmon, R. Sagdeev, Functional imaging and NMR spectroscopy of an operating gas-liquid-solid catalytic reactor, Appl. Catal. A Gen. 267 (1-2) (2004) 143-148. doi:10.1016/j.apcata.2004.02.040.

[7] S. T. Roberts, M. P. Renshaw, M. Lutecki, J. McGregor, A. J. Sederman, M. D. Mantle, L. F. Gladden, Operando Magnetic Resonance: Monitoring the Evolution of Conversion and Product Distribution during the Heterogeneous Catalytic Ethene Oligomerisation Reaction., Chem. Commun. 49 (89) (2013) 10519-21. doi:10.1039/c3cc45896b.

[8] L. Baker, M. Renshaw, M. Mantle, A. Sederman, A. Wain, L. Gladden, Operando magnetic resonance studies of phase behaviour and oligomer accumulation within catalyst pores during heterogeneous catalytic ethene oligomerization, Appl. Catal. A Gen. 557 (2018) 125-134. doi:10.1016/j.apcata.2018.03.011. 
[9] D. Doddrell, D. Pegg, M. Bendall, Distortionless enhancement of NMR signals by polarization transfer, J. Magn. Reson. 48 (2) (1982) 323-327. doi : 10.1016/0022-2364(82)90286-4.

[10] A. J. Sederman, M. D. Mantle, C. P. Dunckley, Z. Huang, L. F. Gladden, In situ MRI study of 1-octene isomerisation and hydrogenation within a trickle-bed reactor, Catal. Letters 103 (1-2) (2005) 1-8. doi:10.1007/ s10562-005-7522-2.

[11] B. S. Akpa, M. D. Mantle, A. J. Sederman, L. F. Gladden, In situ13C DEPT-MRI as a tool to spatially resolve chemical conversion and selectivity of a heterogeneous catalytic reaction occurring in a fixed-bed reactor, Chem. Commun. (21) (2005) 2741. doi:10.1039/b501698c.

[12] C. D'Agostino, T. Kotionova, J. Mitchell, P. J. Miedziak, D. W. Knight, S. H. Taylor, G. J. Hutchings, L. F. Gladden, M. D. Mantle, Solvent Effect and Reactivity Trend in the Aerobic Oxidation of 1,3Propanediols over Gold Supported on Titania: NMR Diffusion and Relaxation Studies, Chem. - A Eur. J. 19 (35) (2013) 11725-11732. doi:10.1002/chem.201300502.

[13] D. Weber, J. Mitchell, J. McGregor, L. F. Gladden, Comparing Strengths of Surface Interactions for Reactants and Solvents in Porous Catalysts Using Two-Dimensional NMR Relaxation Correlations, J. Phys. Chem. C 113 (16) (2009) 6610-6615. doi:10.1021/jp811246j.

[14] C. D'Agostino, J. Mitchell, M. D. Mantle, L. F. Gladden, Interpretation of NMR Relaxation as a Tool for Characterising the Adsorption Strength of Liquids inside Porous Materials, Chem. - A Eur. J. 20 (40) (2014) 13009-13015. doi:10.1002/chem.201403139.

[15] E. Danieli, J. Perlo, B. Blümich, F. Casanova, Small Magnets for Portable NMR Spectrometers, Angew. Chemie Int. Ed. 49 (24) (2010) 4133-4135. doi:10.1002/anie.201000221.

[16] J. Mitchell, L. Gladden, T. Chandrasekera, E. Fordham, Low-field permanent magnets for industrial process and quality control, Prog. Nucl. Magn. Reson. Spectrosc. 76 (2014) 1-60. doi:10.1016/j.pnmrs. 2013. 09.001 . 
[17] B. Blümich, K. Singh, Desktop NMR and Its Applications From Materials Science To Organic Chemistry, Angew. Chemie Int. Ed. 57 (24) (2018) 6996-7010. doi:10.1002/anie.201707084.

[18] M. F. Isaac-Lam, Analysis of Bromination of Ethylbenzene Using a $45 \mathrm{MHz}$ NMR Spectrometer: An Undergraduate Organic Chemistry Laboratory Experiment, J. Chem. Educ. 91 (8) (2014) 1264-1266. doi:10.1021/ed400365p.

[19] J. L. Bonjour, J. M. Pitzer, J. A. Frost, Introducing High School Students to NMR Spectroscopy through Percent Composition Determination Using Low-Field Spectrometers, J. Chem. Educ. 92 (3) (2015) 529533. doi:10.1021/ed500731y.

[20] S. D. Riegel, G. M. Leskowitz, Benchtop NMR spectrometers in academic teaching, TrAC Trends Anal. Chem. 83 (2016) 27-38. doi: $10.1016 / j$.trac.2016.01.001.

[21] E. Danieli, J. Perlo, A. L. L. Duchateau, G. K. M. Verzijl, V. M. Litvinov, B. Blümich, F. Casanova, On-Line Monitoring of Chemical Reactions by using Bench-Top Nuclear Magnetic Resonance Spectroscopy, ChemPhysChem 15 (14) (2014) 3060-3066. doi:10.1002/ cphc.201402049.

[22] G. Guthausen, A. von Garnier, R. Reimert, Investigation of Hydrogenation of Toluene to Methylcyclohexane in a Trickle Bed Reactor by Low-Field Nuclear Magnetic Resonance Spectroscopy, Appl. Spectrosc. 63 (10) (2009) 1121-1127. doi:10.1366/000370209789553156.

[23] M. Goldbach, E. Danieli, J. Perlo, B. Kaptein, V. M. Litvinov, B. Blümich, F. Casanova, A. L. Duchateau, Preparation of Grignard reagents from magnesium metal under continuous flow conditions and on-line monitoring by NMR spectroscopy, Tetrahedron Lett. 57 (1) (2016) 122-125. doi:10.1016/j.tetlet.2015.11.077.

[24] B. Ahmed-Omer, E. Sliwinski, J. P. Cerroti, S. V. Ley, Continuous Processing and Efficient in Situ Reaction Monitoring of a Hypervalent Iodine(III) Mediated Cyclopropanation Using Benchtop NMR Spectroscopy, Org. Process Res. Dev. 20 (9) (2016) 1603-1614. doi: 10.1021/acs.oprd.6b00177. 
[25] K. Singh, E. Danieli, B. Blümich, Desktop NMR spectroscopy for realtime monitoring of an acetalization reaction in comparison with gas chromatography and NMR at 9.4 T, Anal. Bioanal. Chem. 409 (30) (2017) 7223-7234. doi:10.1007/s00216-017-0686-y.

[26] D. Kreyenschulte, E. Paciok, L. Regestein, B. Blümich, J. Büchs, Online monitoring of fermentation processes via non-invasive low-field NMR, Biotechnol. Bioeng. 112 (9) (2015) 1810-1821. doi:10.1002/ bit.25599.

[27] R. Cotts, M. Hoch, T. Sun, J. Markert, Pulsed field gradient stimulated echo methods for improved NMR diffusion measurements in heterogeneous systems, J. Magn. Reson. 83 (2) (1989) 252-266. doi: 10.1016/0022-2364(89)90189-3.

[28] M. Holz, S. R. Heil, A. Sacco, Temperature-dependent self-diffusion coefficients of water and six selected molecular liquids for calibration in accurate 1H NMR PFG measurements, Phys. Chem. Chem. Phys. 2 (20) (2000) 4740-4742. doi:10.1039/b005319h.

[29] S. Bagheri, N. Muhd Julkapli, S. Bee Abd Hamid, Titanium Dioxide as a Catalyst Support in Heterogeneous Catalysis, Sci. World J. 2014 (2014) 1-21. doi:10.1155/2014/727496.

[30] J. R. Zimmerman, W. E. Brittin, Nuclear Magnetic Resonance Studies in Multiple Phase Systems: Lifetime of a Water Molecule in an Adsorbing Phase on Silica Gel, J. Phys. Chem. 61 (10) (1957) 1328-1333. doi: 10.1021/j150556a015.

[31] K. Brownstein, C. Tarr, Spin-lattice relaxation in a system governed by diffusion, J. Magn. Reson. 26 (1) (1977) 17-24. doi:10.1016/ 0022-2364(77) 90230-X.

[32] D. Weber, A. J. Sederman, M. D. Mantle, J. Mitchell, L. F. Gladden, Surface diffusion in porous catalysts, Phys. Chem. Chem. Phys. 12 (11) (2010) 2619. doi:10.1039/b921210h.

[33] M. Leutzsch, M. Falkowska, T.-L. Hughes, A. J. Sederman, L. F. Gladden, M. D. Mantle, T. G. A. Youngs, D. Bowron, H. Myanar, C. Hardacre, An Integrated Total Neutron Scattering - NMR Approach 
for the Study of Heterogeneous Catalysis, Chem. Commun. 54 (72) (2018) 10191-10194. doi:10.1039/c8cc04740e. 